OPEN ACCESS

Edited by:

Sarah Baatout,

Belgian Nuclear Research

Centre, Belgium

Reviewed by:

Roel Quintens,

Belgian Nuclear Research

Centre, Belgium

Kai Rothkamm,

University Medical Center

Hamburg-Eppendorf, Germany

Hubert Thierens,

Ghent University, Belgium

Penny Ann Jeggo,

University of Sussex, United Kingdom

*Correspondence:

Ghazi Alsbeih

galsbeih@kfshrc.edu.sa

Specialty section:

This article was submitted to

Radiation and Health,

a section of the journal

Frontiers in Public Health

Received: 30 December 2020

Accepted: 20 April 2021

Published: 07 June 2021

Citation:

Alsbeih G, Al-Harbi N, Ismail S and Story M (2021) Impaired DNA Repair

Fidelity in a Breast Cancer Patient With Adverse Reactions to Radiotherapy.

Front. Public Health 9:647563.

doi: 10.3389/fpubh.2021.647563

\section{Impaired DNA Repair Fidelity in a Breast Cancer Patient With Adverse Reactions to Radiotherapy}

\author{
Ghazi Alsbeih ${ }^{1,2,3 *}$, Najla Al-Harbi ${ }^{1}$, Sheikh Ismail ${ }^{2,4}$ and Michael Story ${ }^{2,5}$ \\ ${ }^{1}$ Radiation Biology Section, Biomedical Physics Department, King Faisal Specialist Hospital and Research Centre, Riyadh, \\ Saudi Arabia, ${ }^{2}$ Experimental Radiation Oncology Department, University of Texas M.D. Anderson Cancer Center, Houston, \\ TX, United States, ${ }^{3}$ College of Medicine, Alfaisal University, Riyadh, Saudi Arabia, ${ }^{4}$ Commercialization \& Entrepreneurship \\ Department, Texas A\&M University, Bellaire, TX, United States, ${ }^{5}$ Radiation Oncology Department, University of Texas \\ Southwestern Medical Centre, Dallas, TX, United States
}

We tested the hypothesis that differences in DNA double-strand break (DSB) repair fidelity underlies differences in individual radiosensitivity and, consequently, normal tissue reactions to radiotherapy. Fibroblast cultures derived from a radio-sensitive (RS) breast cancer patient with grade 3 adverse reactions to radiotherapy were compared with normal control (NC) and hyper-radiosensitive ataxia-telangiectasia mutated (ATM) cells. DSB repair and repair fidelity were studied by Southern blotting and hybridization to Alu repetitive sequence and to a specific 3.2-Mbp Notl restriction fragment on chromosome 21, respectively. Results for DNA repair kinetics using the Notl fidelity assay showed significant differences $(P<0.001)$ with higher levels of misrepaired (misrejoined and unrejoined) DSBs in RS and ATM compared with NC. At 24-h postradiation, the relative fractions of misrepaired DSBs were 10.64, 23.08, and 44.70\% for NC, RS, and ATM, respectively. The Alu assay showed significant $(P<0.05)$ differences in unrepaired DSBs only between the ATM and both NC and RS at the time points of 12 and $24 \mathrm{~h}$. At $24 \mathrm{~h}$, the relative percentages of DSBs unrepaired were 1.33, 3.43, and $12.13 \%$ for NC, RS, and ATM, respectively. The comparison between the two assays indicated an average of 5-fold higher fractions of misrepaired (Notl assay) than unrepaired (Alu assay) DSBs. In conclusion, this patient with increased radiotoxicity displayed more prominent misrepaired than unrepaired DSBs, suggesting that DNA repair fidelity is a potential marker for the adverse reactions to radiotherapy. More studies are required to confirm these results and further develop DSB repair fidelity as a hallmark biomarker for interindividual differences in radiosensitivity.

Keywords: DNA double-strand breaks, misrepair, Notl fragment, Alu sequence, radiosensitivity, adverse reactions to radiotherapy, repair fidelity, pulsed field gel electrophoresis

\section{INTRODUCTION}

Compelling evidence suggests that adverse reactions to radiotherapy are associated with increased patient sensitivity to ionizing radiation (1). Likewise, individual variation in radiosensitivity is well-recognized and at least partly determined by genetic factors, as clinical, epidemiologic, and laboratory data have indicated (2-6). Initial evidence for 
the heritability of radiosensitivity originated from the studies of rare genetic disorders such as ataxia-telangiectasia (A-T), Nijmegen breakage syndrome, Nijmegen breakage syndromelike disorder (RAD50 deficiency), ligase IV deficiency, A-T-like disorder, and Fanconi's anemia $(7,8)$. Although each syndrome has its own phenotypical characteristics, cells derived from those patients demonstrate spontaneous chromosomal instability and hypersensitivity to ionizing radiation due to mutations affecting DNA strand breaks signaling, recognition, and repair capability (9).

Between the multiple damages produced by ionizing radiation, DNA double-strand breaks (DSBs) are the main critical lesions and are highly consequential for genome integrity (10). Repair is a fundamental inherent mechanism of genome protection, and the ability to rejoin DSBs with appropriate fidelity determines cell fate, recovery, death, or mutagenesis (11). Unrejoined and misrejoined DSBs are important lesions for radiation-induced cell killing, although the relationship between DNA repair, misrepair, and cell survival is not fully understood (12). Misrepaired (unrejoined or misrejoined) DSBs can lead to chromosome aberrations and micronucleus formation, and both endpoints generally correlate with the degree of cell killing (13). However, cellular death mechanisms, cell cycle kinetics, and the various underlying genetic defects influence the expression and detection of chromosome damage, thus making qualitative cytogenetic approaches less precise as quantitative measures of cellular radiosensitivity (14). Another method for examining the fidelity of DNA repair is to measure the ability of cells or cell extracts to reactivate plasmids containing damaged reporter genes. This approach has proven useful for examining DNA repair in some radiosensitive cell lines (15); however, it is also not particularly quantitative, as there was little difference in this measure of DSB repair fidelity between some cell lines with wide differences in radiosensitivity (16).

Another appealing DNA repair fidelity technique that assesses DSB misrepair has been described $(17,18)$. The procedure relies on the use of endonucleases to cleave out specific DNA fragments that are subjected to pulsed-field gel electrophoresis and detected by Southern hybridization to a known probe. Although it is not a widely used technique, the few data obtained using this method were scientifically interesting, as it allowed the detection of differences in the proportion of correctly rejoined DSBs produced by irradiations of different LETs (19) and in the fraction of unrejoined DSBs in cell lines of different origins (20). We have previously used this technique to detect misrepair of radiation-induced DSBs in a patient with unidentified chromosomal fragility syndrome and a family history of radiosensitivity (21). Here, we extend this basic research work to examine the ability of this technique to detect differences in DSB repair fidelity in a fibroblast cell line derived from a breast cancer patient who developed marked late adverse reactions to radiotherapy. The results were compared with a cell line of a patient with no radiotherapy tissue reactions and an extremely radiosensitive A-T mutated (ATM) cell line.

\section{MATERIALS AND METHODS Cell Cultures, Patients, and Ethical Considerations}

Three primary non-transformed human skin fibroblast cultures, normal control (NC), radiosensitive (RS), and ATM were used. The ethics committee of the institutional review board approved the study (CA-06294/16672/50192; 1990). The ATM cell line (GM01588A) was purchased from the American Type Culture Collection (Manassas, VA, USA). The NC and RS were derived from two breast cancer patients, as described previously (22). Briefly, the NC patient did not develop any discernable (grade 0; Radiation Therapy Oncology Group and the European Organization for Research and Treatment of Cancer grading system) adverse effects, whereas the RS patient developed marked (grade 3) skin atrophy and telangiectasia. Both patients were treated by definitive radiotherapy (50 Gy in 2 Gy fractions). Compared with NC, RS was considered to have excessive late reactions for the dose received, which was verified from their treatment and dosimetry records. The cumulative total dose delivered to different normal tissues was estimated from computed tomography treatment plans. Late effects were documented from patient records. The median follow-up was 19 months (range: 13 to 25) at the time of data collection. The in vitro radiosensitivity characterization of the cell strains using clonogenic survival curves was published previously $(23,24)$. Briefly, the surviving fraction at $2 \mathrm{~Gy}$ radiation dose was 0.34 [95\% confidence interval $(\mathrm{CI})=$ $0.31-0.37$ ], 0.18 (95\% CI $=0.13-0.25)$, and 0.03 (95\% CI $=0.02-0.04)$ for NC, RS, and ATM cell strains, respectively. The three cell lines were maintained in alpha minimal essential medium supplemented with $15 \%$ fetal bovine serum. Experiments were performed with contact-inhibited cultures to minimize cell cycle-dependent variations in DNA repair. All incubations were performed at $37^{\circ} \mathrm{C}$ in a humidified atmosphere of $5 \%$ carbon dioxide.

\section{Irradiation}

Cells were irradiated on ice in $150-\mathrm{mm}$ Petri dishes using a ${ }^{137} \mathrm{Cs}$ source at a dose rate of $3.65 \mathrm{~Gy} / \mathrm{min}$.

\section{Notl and Alu DNA Double-Strand Breaks Repair Assays}

The NotI repair fidelity assay involves the use of endonuclease to cleave out a specific DNA fragment that can be detected by Southern hybridization to a known probe. Using the NotI rare cutting restriction enzyme, a unique 3.2-Mbp restriction fragment is cleaved out of the long arm of chromosome 21. After subjecting DNA to pulsed-field gel electrophoresis, the NotI 3.2-Mbp fragment migrates as a single band, which is detected and quantified by hybridization to the D21S1 single copy probe. Unrejoined and misrejoined NotI fragments induced by irradiation migrate separately from the NotI band. The extent of incomplete restoration of the 3.2-Mbp Not I band is taken as a quantitative measure of misrepair in this specific region of the genome. The Alu assay follows the same principal except for the 
endonuclease use; being a highly repetitive sequence, it assesses unrepaired DSBs in the whole genome.

The Southern blot procedures for NotI and Alu DNA repair assays were published in detail previously $(21,25)$. Briefly, confluent cells were irradiated with either $30 \mathrm{~Gy}$ (for Alu genomic probe) or $80 \mathrm{~Gy}$ (for NotI specific fragment probe) and incubated at $37^{\circ} \mathrm{C}$ for up to $24 \mathrm{~h}$. The choice of radiation doses was determined in preliminary experiments and optimized to induce DSBs in approximately $80 \%$ of the target DNA in each of the Alu and NotI fragments (21). The cells were trypsinized and centrifuged. The pellet was resuspended at a concentration of $2-4 \times 10^{7}$ cells $/ \mathrm{mL}$, for NotI DSBs repair fidelity, or $10^{5}$ cells/mL for Alu total genomic DSB repair assays. The cell suspension was mixed with $1 \%$ low-melting point agarose (InCert, FMC BioProducts) and poured into plastic molds. Solidified plugs were lysed [0.5-M ethylenediaminetetracetic acid disodium $\left(\mathrm{Na}_{2} \mathrm{EDTA}\right), 1 \%$ sodium lauroyl sarcosine, 1 $\mathrm{mg} / \mathrm{mL}$ proteinase $\mathrm{K}, \mathrm{pH}$ 8] at $50^{\circ} \mathrm{C}$ for $2-3$ days, washed and stored in $0.5-\mathrm{M} \mathrm{Na} 2 \mathrm{EDTA}(\mathrm{pH} 8)$ at $4^{\circ} \mathrm{C}$. For restriction enzyme digestion, DNA in a half-plug was digested with 25 units of NotI restriction enzyme (Promega, Madison, WI, USA) at $37^{\circ} \mathrm{C}$ overnight and inactivated at $50^{\circ} \mathrm{C}$ for $2 \mathrm{~h}$. As for genomic DNA repair $(\mathrm{Alu})$, there is no need for a restriction enzyme treatment.

DNA DSBs were separated by pulsed-field gel electrophoresis, using a CHEF Mapper or CHEF-DR II electrophoresis system (Bio-Rad), in a $0.5 \times$ Tris/borate/EDTA buffer ( 45 -mM Tris-base, 45-mM boric acid, 1-mM Na 2 EDTA; $\mathrm{pH}$ 8). The field strength was $1.5 \mathrm{~V} / \mathrm{cm}$ with pulse times linearly increasing from 50 to $5,000 \mathrm{~s}$. Electrophoresis was carried out for $18 \mathrm{~h}$ at $24^{\circ} \mathrm{C}$ for total genomic DNA (Alu assay) and at $12^{\circ} \mathrm{C}$ for $140 \mathrm{~h}$ for DNA repair fidelity (NotI assay). Gels were stained with ethidium bromide for $15 \mathrm{~min}$, destained for $1 \mathrm{~h}$, and photographed with a digital camera system under ultraviolet transillumination. Schizosaccharomyces pombe and Hansenula wingei chromosomes served as size markers. For DNA transfer, gels were exposed to a germicidal ultraviolet lamp and soaked in the alkaline transfer solution $(0.4-\mathrm{M} \mathrm{NaOH} ; 0.6-\mathrm{M} \mathrm{NaCl})$ for $30 \mathrm{~min}$, and the DNA was transferred by capillary action to nylon membranes (GeneScreen Plus, Du Pont, NEN Research Products, Boston, MA, USA) over 2 days and air-dried.

For hybridization to the 3.2-Mbp NotI restriction fragment, the plasmid containing the D21S1 probe (pPW228C) was isolated from the host bacteria; the insert was cut out with EcoRI and gel-purified by standard procedures. Radioactively labeled probe was produced via random priming kit (Boehringer Mannheim, Gaithersburg, MD, USA) using $\left[\alpha-{ }^{32} \mathrm{P}\right] \mathrm{dCTP}(222 \mathrm{TBq} / \mathrm{mmol}$, NEN Life Science Products, Boston, USA). The membranes were hybridized for 2-2.5 days at $45^{\circ} \mathrm{C}$ in Hybrisol I (Intergen, Burlington, MA, USA) and heat-denatured ${ }^{32}$ P-labeled DNA probe $\left(0.3-1 \times 10^{8} \mathrm{cpm} /\right.$ membrane). The membranes were washed and exposed to storage phosphor screens for 1 to 6 days, depending on the signal intensity of each membrane. Likewise, the Alu probe was prepared and processed in a similar way except that it was cut out of hosting plasmid (BLUR8) using Bamh1 restriction enzyme, and the labeled probe was hybridized overnight. At least two experiments were carried out for each cell line, two samples from each experiment were run on separate gels, and the results were pooled.

\section{Data Analysis}

The quantitative analysis of the Southern blot data was described previously (21). Briefly, the total intensity $\left(I_{0}\right)$ was calculated as the integral of the signal in the whole lane after baseline adjustment. For DNA repair in the whole genome (Alu assay), the intensity of the migrating DSBs in the Alu sequence $\left(I_{1}\right)$ was quantified by the integral of the signal below the well. The relative amount of DNA released from the plugs (corresponding to DSBs $\leq 10-12 \mathrm{Mbp}$ ) was calculated by dividing the signal intensity of the migrating DNA $\left(I_{1}\right)$ by that of the whole lane, including the wells $\left(I_{0}\right)$. For DSB repair fidelity (NotI assay), the intensity of the full-length NotI fragments $(I)$ was quantified by the integral of the signal in the 3.2-Mbp band. The signal intensity of misrepaired (misrejoined and unrejoined) DSBs $\left(I_{1}\right)$ was calculated by subtracting $I$ from $I_{0}$. The relative amount of misrepaired 3.2-Mbp NotI restriction fragment in each lane was calculated by dividing $I_{1}$ by $I_{0}$. For both assays, the kinetics of DSB repair, after various repair times, was presented as a fraction (\%) of DSBs remaining unrepaired or misrepaired (also known as the fraction of "radiation" activity released). The DSBs remaining unrepaired (Alu assay) or misrepaired (NotI assay) were calculated by dividing the relative amount by that induced before any repair ( $0 \mathrm{~h}$ repair time) after subtraction of background (0 Gy) for each. Testing for statistically significant differences in the kinetics of DSBs misrepaired or remaining unrepaired between the cell strains was carried out using the one-way repeated measures analysis of variance test. This test compares differences in the mean values, computed over all the repair time points, among the cell strains. Comparing between the cell strains at each time point of repair was carried out using the one-way analysis of variance test. Testing for the difference from baseline zero-level was conducted using a one-tailed $t$-test. SigmaPlot software (versions 12.5 and 13; Systat Software Inc., San Jose, CA, USA) was used for statistical analysis. $P<0.05$ was considered significant.

\section{RESULTS}

Figure 1 shows representative examples of ethidium bromidestained gels (left panels) and the corresponding membranes hybridized to the Alu (BLUR8) and NotI (D21S1) probes (right panels). On the gel photographs, we can distinguish the wells containing non-migrating high molecular weight DNA and a smear of migrating DSBs of approximately $5.7 \mathrm{Mbp}$ or less. On the membranes, we only see the DNA hybridized with the probes. The Alu genomic probe hybridizes to the widespread Alu sequence in the human genome. The signal below the wells (plugs) represents the amount of DSBs in the whole genome. The D21S1 probe hybridizes to a specific 3.2-Mbp NotI fragment on chromosome 21. Full-length NotI fragments are located in a band at 3.2 Mbp. Irradiation breaks down the NotI fragments leading to a smear seen below the NotI band. When the time for repair increases from 0 to $24 \mathrm{~h}$, the broken DSBs are repaired, resulting 


\section{A Alu total genomic DNA DSB repair:}

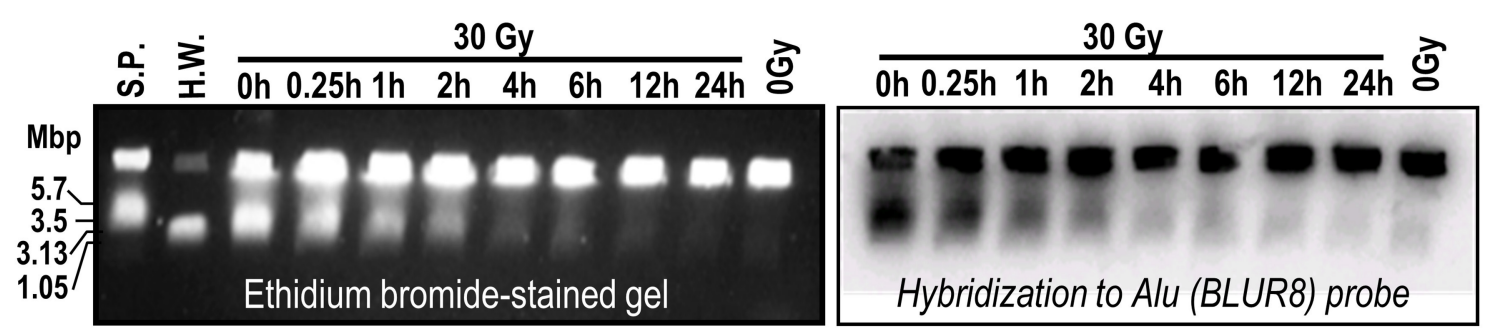

в NotI DNA DSB repair fidelity assay:

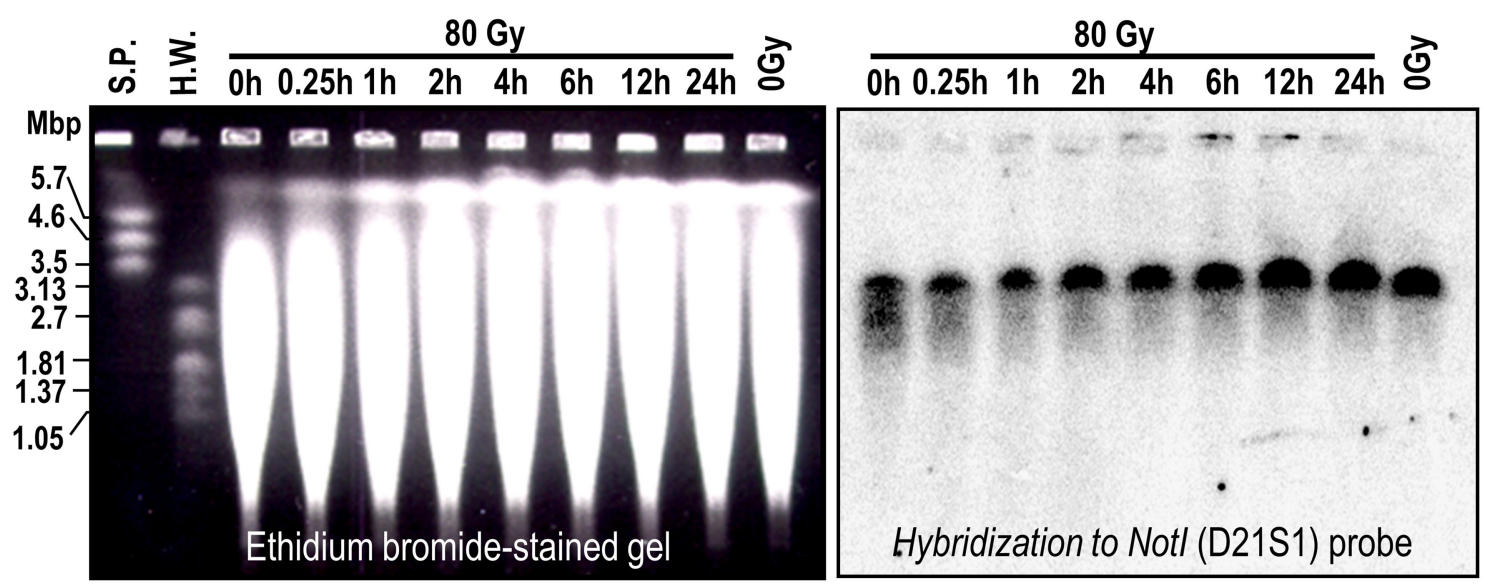

FIGURE 1 | Representative ethidium bromide-stained gels and corresponding membranes hybridized to Alu repetitive sequence in whole genome (BLURB probe; A) or specific Notl fragments located on chromosome 21 (D21S1 probe; B). Bulk rejoining of DSBs in genomic DNA and DSB repair fidelity in a 3.2-Mbp DNA fragment. Data from NC cell strain. S.P. (S. pombe) and H.W. (H. wingei) are DNA size standards.

in the gradual diminishing of the Alu signal below the wells and restitution of the full-length NotI fragments.

Illustrative comparison of Alu and NotI hybridized membranes in the three cell lines are shown in the upper panels of Figure 2. With increasing time for repair, the hybridization signals were different between the cell lines. The control cell line showed the highest restitution of DSBs compared with the radiosensitive patient and particularly the extremely sensitive ATM cell strain that showed a comparatively high level of unrepaired DSBs. Although the average background level of DSBs (0 Gy) was 7.31\% [range: 6.48-9.36\%; standard deviation $(\mathrm{SD})=1.79$ ] in the Alu assay, it was $27.47 \%$ (range: $21.76-$ $35.28 \%$; $\mathrm{SD}=6.99$ ) in the NotI assay. In the Alu whole-genome assay, the fraction of induced DSBs, without repair, after a dose of $30 \mathrm{~Gy}$ was $0.84(\mathrm{SD}=0.02), 0.87(\mathrm{SD}=0.02)$, and $0.83(\mathrm{SD}=$ 0.03 ) for NC, RS, and ATM, respectively. Similarly, in the NotI fragments, a dose of $80 \mathrm{~Gy}$ induced comparable fractions in the cell strains [NC: $0.79(\mathrm{SD}=0.04)$, RS: 0.83 ( $\mathrm{SD}=0.05)$, and ATM: $0.84(\mathrm{SD}=0.03)]$.

The results for DSBs remaining unrepaired or misrepaired after subtraction of $0 \mathrm{~Gy}$ and normalizing to the induction level $(0 \mathrm{~h})$ are presented in the lower panels of Figure 2. The repair kinetics indicated differences not only between the three cell strains but also between genomic Alu and specific NotI repair assays. Although it is obvious that these cell lines display a wide range of radiosensitivity exhibited by different capacities to restitute broken DNA, the NotI repair fidelity assay showed higher levels of misrepaired DSBs. For example, at $24 \mathrm{~h}$ of repair, where the largest differences are seen, the average values for residual DSBs were 5.60\% $(\mathrm{SD}=5.73)$ for $\mathrm{Alu}$ and $26.13 \%$ $(\mathrm{SD}=17.25)$ for NotI assays. This is an average of a 5 -fold increase in residual DSBs detection in the DNA repair fidelity assay compared with general or bulk DNA repair. In addition, using a one-tailed $t$-test to examine the divergence of the 24-h residual DSBs in the three cell strains from the baseline (0\%) level, the differences for the NotI assay were statistically significant $(P$ $=0.03)$, although not so for the Alu assay $(P=0.08)$.

The percentage of DSBs misrepaired or remaining unrepaired in the Not I fragments after $24 \mathrm{~h}$ of repair were 10.64, 23.08, and $44.70 \%$ for NC, RS, and ATM, respectively. This indicates a 2.2- and 4.2-fold increase in misrepair in RS and ATM as compared with NC, respectively. Statistical analysis of Not I DNA repair kinetics showed a significant difference in DSBs misrepaired between the three cell strains (one-way repeated measures analysis of variance, $P<0.001$ ). In comparison, the $A l u$ assay showed lower levels of DSBs remaining unrepaired. The NC and RS showed similar repair kinetics, whereas ATM displayed a relatively higher level of unrepaired DSBs. The percentages of 

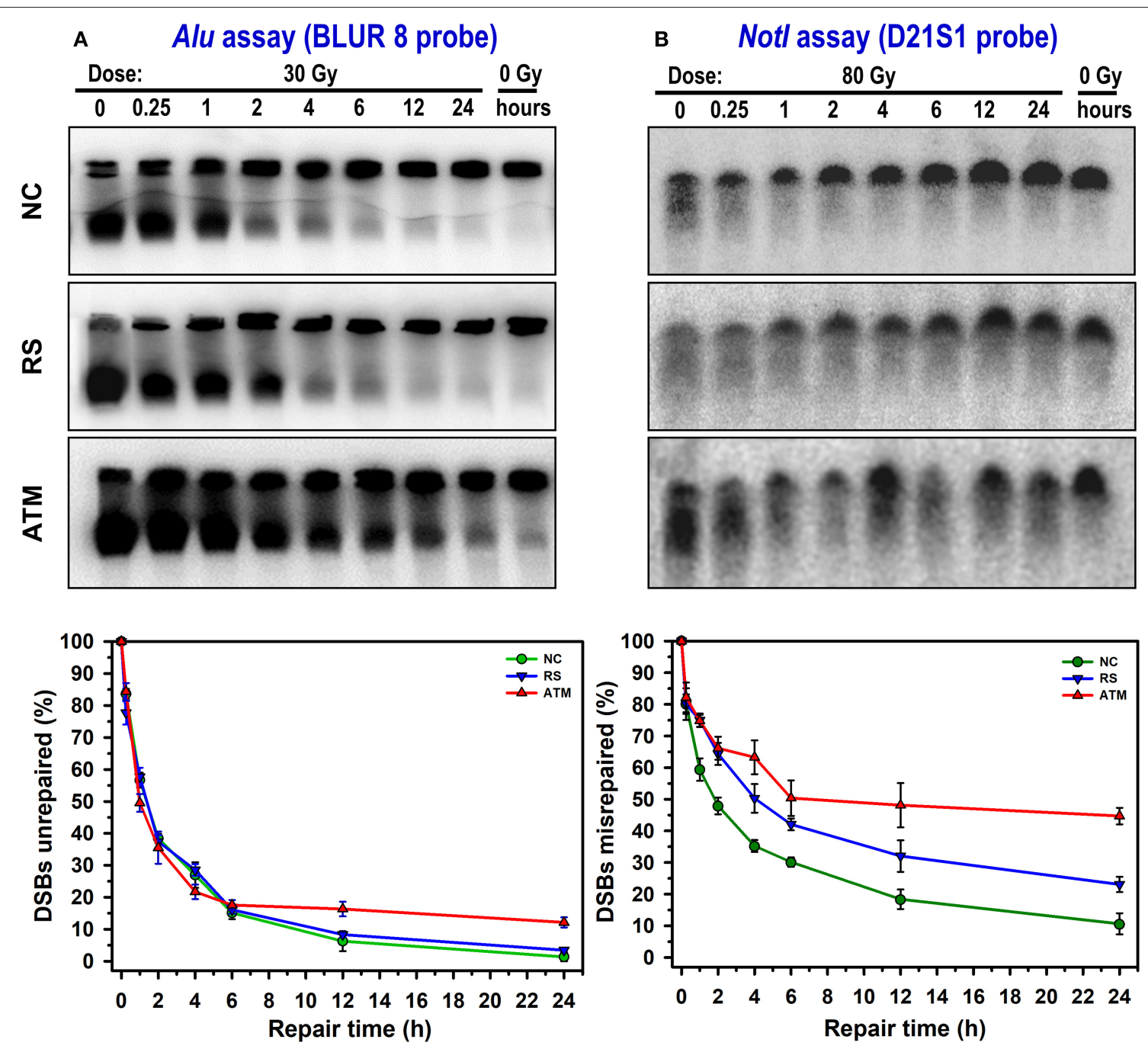

FIGURE 2 | Representative examples of DSB repair and repair fidelity in NC, RS, and ATM cell strains. Membranes were hybridized to Alu repetitive sequence (whole-genome DSBs unrepaired; A) and specific Notl fragments (DSBs misrepaired; B). Curves show corresponding kinetics of DSB repair after subtraction of membrane background and normalizing to total amount of DSBs induced immediately after irradiation (0-h repair time). Data points represent mean, and error bars represent standard error of mean. Results of statistical analysis (one-way repeated measures analysis of variance) are as follows: Notl assay, there is an overall significant difference between cell lines $(P<0.001)$. Pairwise comparison: ATM vs. NC: $P<0.001$; RS vs. NC: $P=0.014$; ATM vs. RS: $P=0.034$. For Alu assay, no overall significant differences between cell lines $(P=0.82)$. However, significant differences (one-way analysis of variance) between cell lines $(P<0.05)$ were observed at 12 and $24 \mathrm{~h}$. Pairwise comparison at $12 \mathrm{~h}$ : ATM vs. NC: $P=0.024$; ATM vs. RS: $P=0.054$; RS vs. NC: $P=0.548$. Pairwise comparison at 24 h: ATM vs. NC: $P<$ $0.001 ;$ ATM vs. RS: $P<0.001$; RS vs. NC: $P=0.287$.

the DSBs unrepaired at $24 \mathrm{~h}$ in the $A l u$ whole-genome assay were $1.33,3.43$, and $12.13 \%$ for NC, RS, and ATM, respectively. This indicates a 2.6- and 9.3-fold increase in DSBs unrepaired in RS and ATM as compared with NC, respectively. Nevertheless, there was no statistically significant difference in unrepaired DSBs between the three cell strains when all time points are considered together (one-way repeated measures analysis of variance, $P=$ 0.82). However, testing for dissimilarities at each time point of repair kinetics revealed statistically significant differences at 12 and $24 \mathrm{~h}$ between ATM and both NC and RS (one-way analysis of variance, $P<0.05$ ); meanwhile, no difference was observed between NC and RS (Figure 2).

\section{DISCUSSION}

In this study, we examined the possibility of detecting differences in DSB misrejoining (unrepaired and misrepaired) in a primary human fibroblast strain derived from a clinically radiosensitive patient with marked (grade 3 ) adverse effects to radiotherapy. The results were compared with a cell line from a patient who exhibited no adverse effects (grade 0 ) and a cell line derived from an ATM patient who is known for their extreme radiosensitivity (patients typically develop grade 4 adverse reactions). In the three cell lines, a dose of $30 \mathrm{~Gy}$ led to the induction (before any repair) of comparable amounts of DSBs in the entire genome (Alu probe, 
$0.85, \mathrm{SD}=0.02)$. Similarly, a dose of $80 \mathrm{~Gy}$ produced comparable amounts of DSBs in the NotI fragments $(0.81, \mathrm{SD}=0.02)$. These results are in line with results obtained by other investigators (26). However, when irradiated cells were allowed to repair, these different cell lines displayed a distinguishable capacity to join DSB ends (Figure 2). The repair kinetics displayed an exponential decay shape with an initial rapid decrease in the fraction of DSBs misrepaired or unrepaired that extends to approximately $4 \mathrm{~h}$, followed by a slight decrease after that. This shape is quite common for DSBs repair kinetics, which can be mathematically fitted by first-order or biphasic exponential decay equations $(27,28)$. Although such a fitting may facilitate analysis and contribute to the characterization of hypothetical biological processes, it is not required to derive conclusions from the results presented (29).

The three cell strains showed comparable initial repair kinetics for the entire genome (Alu assay), whereas differences were mainly detectable in the slow distal time points from 12 to $24 \mathrm{~h}$ (Figure 2). The fraction of DSBs remaining unrepaired was between 1 and $12 \%$ of the initial damage (i.e., 88 to $99 \%$ were rejoined). However, this level does not seem to significantly diverge from the baseline $(0 \%)$ level $(P=0.08)$. Note that the $\mathrm{NC}$ and the RS cell lines showed similar kinetics when compared with ATM, which seemed to have a slightly faster repair up to $4 \mathrm{~h}$, followed by slower repair after that. Although there were no overall significant differences between the three cell strains $(P=0.82)$, the ATM showed, as expected, significantly $(P<$ $0.05)$ higher unrepaired DSBs at 12 and $24 \mathrm{~h}$ postradiation. In contrast, for the repair fidelity assay, the three cell strains showed distinguishable kinetic curves of statistical significance $(P<$ 0.001 ) at much earlier repair times, which continued to $24 \mathrm{~h}$. On average, the NotI assay showed a 5-fold higher level of residual DSBs than the Alu assay. Therefore, the repair fidelity assay was able to detect differences in DSB repair between patients with and without radiotoxicities and the extreme radiosensitivity of ATM. This is consistent with a previous observation of normal, ATM, and AT-heterozygous cell types (26). The NC cell line exhibited the lowest rate of misrepair (highest repair fidelity) in the 3.2Mbp NotI restriction fragment with a level of misrejoining and unrejoining at $24 \mathrm{~h}$ of $11 \%$ of the initial damage, i.e., $89 \%$ of ends were rejoined. The ATM cell line showed a relatively high level of misrepair, $45 \%$ (55\% of rejoining), whereas the RS cell line was intermediate with $23 \%$ ( $77 \%$ rejoining) misrepair. The latter is a relatively lower misrepair in comparison with a previously reported radiosensitive cell strain, which showed a NotI DSBs misrepair level at $24 \mathrm{~h}$ of $37 \%$ (21). Furthermore, these levels of DSB misrepair at $24 \mathrm{~h}$ seem to significantly diverge from the baseline $(0 \%)$ level $(P=0.03)$.

The results suggest that, for the radiosensitive patient, misrepair of broken DNA ends in non-transformed fibroblasts is associated with late normal tissue reactions after radiotherapy. In view of the results with a normally sensitive patient, a radiosensitive patient who developed late adverse complications, and an ATM patient (GM01588), who will invariably develop severe and even fatal complications if treated with a standard radiotherapy regimen, it is tempting to speculate that the misrepair assay could provide a better resolution and more accurate measure of radiosensitivity than DNA repair within the entire genome. Nevertheless, a previous work using the Not $\mathrm{I}$ assay identified differences in misrepair only after fractionated irradiation (26). Using normal (derived from a healthy volunteer with no radiosensitivity data), ATM, and AT heterozygous cell lines, differences were observed after low dose per fraction (5 and $10 \mathrm{~Gy})$ and not with a high $(20 \mathrm{~Gy}$ ) or at the total $80 \mathrm{~Gy}$ given as a single dose. In our study, the variation was observed after a single 80-Gy dose. Other doses were not examined. The apparent discrepancy may lay in the levels of misrejoined DSB observed in the normal cell strains. However, the dissimilarities in the methodological details between the two studies preclude a precise comparison.

Although this study is not designed to delve into the particular radiosensitivity of the ATM cell strain, it can be speculated from the data that radiosensitivity is associated with an increased level of misrepaired DSBs. This is in line with the observation that it displayed increased levels of unrepaired DSBs (30). The present demonstration of a higher level of misrejoining observed in the NotI repair fidelity assay (Figure 2) supports a mechanistic answer for the increased rate of chromosomal aberrations in ATM and other chromosomal fragility syndromes (24). Genome editing technology demonstrated that artificially introduced heterozygous mutations of the ATM gene increased the number of chromosomal aberrations after irradiation and shed light on the genetic basis underlying individual differences in radiosensitivity within the human population (31). In addition, researchers described ATM cells as having higher initial repair speed followed by a stagnant slow repair leading to higher DSBs unrepaired in the whole genome (30), which can be perceived in this study (Figure 2, left panels). However, this is not the case in the repair fidelity assay, which suggests slower initial repair kinetics than that observed with NC and RS cell strains (Figure 2, right panels).

Finally, this study contributes to the ever-expanding experimental evidence for the increased radiosensitivity of a small percentage of radiotherapy patients treated with standard regimens. Those patients develop adverse radiation sequelae that cannot be attributed to dose distribution or volume irradiation (22). Many reports about cohorts and individual cases of radiosensitivity have been published where clinical radiosensitivity was associated with certain in vitro experimental endpoints with variable results (14). Between the various mechanistic pathways investigated, the radiation-induced DNA damage response remains the most well-characterized $(32,33)$. However, some other mechanisms and pathways were suggested to be involved in patient radiosensitivity, including oxidative stress, stem cell response, activation of inflammation pathways with the secretion of cytokines, genetics, non-coding RNA, and potentially epigenetic factors that can be studied using a large number of functional assays (3). For instance, individual variations in radiosensitivity have been attributed to a dissimilar genetic makeup affecting various cellular processes (2). However, besides a few syndromes with identified genetic mutations, the culprit molecular pathway affecting the radiosensitivity of phenotypically normal patients remains elusive (34). For mildly and moderate overreacting patients, polymorphic 
genetic variations in DNA repair and other processes are believed to be related to the interindividual reactions to radiotherapy $(35,36)$.

The genomic basis of radiosensitivity is important both in cancer therapy, where normal and tumor cells differ in their response to treatment (37), and in neoplastic transformation, where exposure to radiation (as in occupational and diagnostic radiology) and environmental hazards may have different carcinogenic susceptibilities in the population (38). In fact, DSB repair fidelity is believed to be an important mechanism for radiation-induced cancer and a potential marker for radiation susceptibility. However, both NC and RS are breast cancer patients, and ATM is known to predispose to a certain type of malignancies. Thus, the lowest residual level of DSB misrepaired observed in the NC cells could reflect cancer susceptibility in this breast cancer patient with normal radiosensitivity. Furthermore, the striking observation of the larger amount of misrepaired DSBs in the NotI repair fidelity assay compared with the modest amount of unrepaired DSBs in the whole genome (Alu assay) suggests that DNA repair fidelity is also a mechanism involved in radiation sensitivity. Therefore, it can be speculated that assessing DNA repair fidelity in cancer patients may be a useful indicator as a prognostic or predictive marker for the likelihood of developing radiotoxicity after radiation treatment or exposure to other clastogenic agents.

\section{CONCLUSIONS}

This study demonstrated that in the cells derived from this patient who had severe adverse reactions to radiotherapy, DNA DSBs were more likely to be misrepaired rather than unrepaired. This may imply that DNA repair fidelity is a mechanism leading to adverse reactions to radiotherapy. More studies with large patients' cohort are required to confirm these results. The DNA

\section{REFERENCES}

1. Story M, Ding LH, Brock WA, Ang KK, Alsbeih G, Minna J, et al. Defining molecular and cellular responses after low and high linear energy transfer radiations to develop biomarkers of carcinogenic risk or therapeutic outcome. Health Phys. (2012) 103:596-606. doi: 10.1097/HP.0b013e3182692085

2. Rattay T, Talbot CJ. Finding the genetic determinants of adverse reactions to radiotherapy. Clin Oncol. (2014) 26:301-8. doi: 10.1016/j.clon.2014.02.001

3. Averbeck D, Candeias S, Chandna S, Foray N, Friedl AA, Haghdoost S, et al. Establishing mechanisms affecting the individual response to ionizing radiation. Int J Radiat Biol. (2020) 96:297-323. doi: 10.1080/09553002.2019.1704908

4. Seibold P, Auvinen A, Averbeck D, Bourguignon M, Hartikainen JM, Hoeschen C, et al. Clinical and epidemiological observations on individual radiation sensitivity and susceptibility. Int J Radiat Biol. (2020) 96:32439. doi: 10.1080/09553002.2019.1665209

5. Zyla J, Kabacik S, O'Brien G, Wakil S, Al-Harbi N, Kaprio J, et al. Combining CDKN1A gene expression and genome-wide SNPs in a twin cohort to gain insight into the heritability of individual radiosensitivity. Funct Integr Genomics. (2019) 19:575-85. doi: 10.1007/s10142-019-00658-3

6. Bachelet J-T, Granzotto A, Ferlazzo M, Sonzogni L, Berthel E, Devic C, et al. First radiobiological characterization of skin and bone cells from a patient suffering from the PI3KCA-Related Overgrowth Spectrum (PROS) syndrome. Arch Clin Med Case Rep. (2020) 4:1052-66. doi: 10.26502/acmcr.96550297 repair fidelity assay may be an important endpoint to be further perused and developed as a hallmark for radiosensitivity.

\section{DATA AVAILABILITY STATEMENT}

The original contributions generated for this study are included in the article/supplementary material, further inquiries can be directed to the corresponding author/s.

\section{ETHICS STATEMENT}

The studies involving human participants were reviewed and approved by Ethics Committee of the Institutional Review Board (CA-06294/16672/50192). The patients/participants provided their written informed consent to participate in this study.

\section{AUTHOR CONTRIBUTIONS}

MS and GA: conceptualization and funding acquisition. GA and SI: methodology. GA, SI, and NA-H: investigation. GA: formal analysis. MS and GA: validation. GA: writing the original draft. All authors: review and editing.

\section{FUNDING}

We acknowledge the support of the National Cancer Institute Grants CA-06294, CA-16672, and CA-50192 and King Faisal Specialist Hospital and Research Center Grant RAC\# 2000031.

\section{ACKNOWLEDGMENTS}

We thank Professor Williams Brock for his leadership, Dr. Fady Geara for providing the cell strains, and Dr. Khaled Al-Hadyan and Ms. Sherri Bergh for their assistance.

7. Gatti RA. The inherited basis of human radiosensitivity. Acta Oncol. (2001) 40:702-11. doi: 10.1080/02841860152619115

8. Zahnreich S, Weber B, Rosch G, Schindler D, Schmidberger H. Compromised repair of radiation-induced DNA double-strand breaks in Fanconi anemia fibroblasts in G2. DNA Repair. (2020) 96:102992. doi: 10.1016/j.dnarep.2020.102992

9. Alsbeih G, Al-Meer RS, Al-Harbi N, Bin Judia S, Al-Buhairi M, Venturina NQ, et al. Gender bias in individual radiosensitivity and the association with genetic polymorphic variations. Radiother Oncol. (2016) 119:23643. doi: 10.1016/j.radonc.2016.02.034

10. Iliakis G, Mladenov E, Mladenova V. Necessities in the processing of DNA double strand breaks and their effects on genomic instability and cancer. Cancers (Basel). (2019) 11:1671. doi: 10.3390/cancers11111671

11. Chatterjee N, Walker GC. Mechanisms of DNA damage, repair, and mutagenesis. Environ Mol Mutagen. (2017) 58:235-63. doi: 10.1002/em.22087

12. McMahon SJ, Schuemann J, Paganetti H, Prise KM. Mechanistic modelling of DNA repair and cellular survival following radiation-induced DNA damage. Sci Rep. (2016) 6:33290. doi: 10.1038/srep33290

13. Durante M, Formenti SC. Radiation-induced chromosomal aberrations and immunotherapy: micronuclei, cytosolic DNA, and interferon-production pathway. Front Oncol. (2018) 8:192. doi: 10.3389/fonc.2018.00192

14. Habash M, Bohorquez LC, Kyriakou E, Kron T, Martin OA, Blyth BJ. Clinical and functional assays of radiosensitivity and radiation-induced second cancer. Cancers (Basel). (2017) 9:147. doi: 10.3390/cancers9110147 
15. Hannan MA, Siddiqui Y, Rostom A, Al-Ahdal MN, Chaudhary MA, Kunhi M. Evidence of DNA repair/processing defects in cultured skin fibroblasts from breast cancer patients. Cancer Res. (2001) 61:3627-31.

16. Collis SJ, Sangar VK, Tighe A, Roberts SA, Clarke NW, Hendry JH, et al. Development of a novel rapid assay to assess the fidelity of DNA double-strand-break repair in human tumour cells. Nucleic Acids Res. (2002) 30:E1. doi: 10.1093/nar/30.2.e1

17. Lobrich M, Ikpeme S, Kiefer J. DNA double-strand break measurement in mammalian cells by pulsed-field gel electrophoresis: an approach using restriction enzymes and gene probing. Int J Radiat Biol. (1994) 65:62330. doi: 10.1080/09553009414550731

18. Lobrich M, Rydberg B, Cooper PK. Repair of X-ray-induced DNA doublestrand breaks in specific NotI restriction fragments in human fibroblasts: joining of correct and incorrect ends. Proc Natl Acad Sci U S A. (1995) 92:12050-4. doi: 10.1073/pnas.92.26.12050

19. Kuhne M, Rothkamm K, Lobrich M. No dose-dependence of DNA doublestrand break misrejoining following alpha-particle irradiation. Int J Radiat Biol. (2000) 76:891-900. doi: 10.1080/09553000050050909

20. Rothkamm K, Lobrich M. Misrejoining of DNA double-strand breaks in primary and transformed human and rodent cells: a comparison between the HPRT region and other genomic locations. Mutat Res. (1999) 433:193205. doi: 10.1016/S0921-8777(99)00008-7

21. Alsbeih G, Brock W, Story M. Misrepair of DNA double-strand breaks in patient with unidentified chromosomal fragility syndrome and family history of radiosensitivity. Int $J$ Radiat Biol. (2014) 90:53-9. doi: 10.3109/09553002.2014.859764

22. Geara FB, Peters LJ, Ang KK, Wike JL, Brock WA. Prospective comparison of in vitro normal cell radiosensitivity and normal tissue reactions in radiotherapy patients. Int J Rad Oncol Biol Phys. (1993) 27:11739. doi: 10.1016/0360-3016(93)90540-C

23. Geara FB, Peters LJ, Ang KK, Wike JL, Sivon SS, Guttenberger R, et al. Intrinsic radiosensitivity of normal human fibroblasts and lymphocytes after high- and low-dose-rate irradiation. Cancer Res. (1992) 52:6348-52.

24. Alsbeih G, Story MD, Maor MH, Geara FB, Brock WA. Chromosomal fragility syndrome and family history of radiosensitivity as indicators for radiotherapy dose modification. Radiother Oncol. (2003) 66:3414. doi: 10.1016/S0167-8140(02)00327-4

25. Alsbeih G, Brock WA, Terry N, Story MD. Analysis of radiationinduced DNA double-strand breaks misrepair is not compromized by broken DNA in human fibroblasts. Radiat Environ Biophys. (2003) 42:10711. doi: 10.1007/s00411-003-0197-4

26. Lobrich M, Kuhne M, Wetzel J, Rothkamm K. Joining of correct and incorrect DNA double-strand break ends in normal human and ataxia telangiectasia fibroblasts. Genes Chromosomes Cancer. (2000) 27:59-68.

27. Foray N, Fertil B, Alsbeih MG, Badie C, Chavaudra N, Iliakis G, et al. Dose-rate effect on radiation-induced DNA double-strand breaks in the human fibroblast HF19 cell line. Int J Radiat Biol. (1996) 69:2419. doi: 10.1080/095530096146084

28. Plante I, Slaba T, Shavers Z, Hada M. A bi-exponential repair algorithm for radiation-induced double-strand breaks: application to simulation of chromosome aberrations. Genes (Basel). (2019) 10:936. doi: 10.3390/genes10110936

29. Wang H, Zeng ZC, Bui TA, Sonoda E, Takata M, Takeda S, et al. Efficient rejoining of radiation-induced DNA double-strand breaks in vertebrate cells deficient in genes of the RAD52 epistasis group. Oncogene. (2001) 20:221224. doi: 10.1038/sj.onc. 1204350

30. Foray N, Priestley A, Alsbeih G, Badie C, Capulas EP, Arlett CF, et al. Hypersensitivity of ataxia telangiectasia fibroblasts to ionizing radiation is associated with a repair deficiency of DNA double-strand breaks. Int J Radiat Biol. (1997) 72:271-83. doi: 10.1080/095530097143266

31. Miyamoto T, Akutsu SN, Tauchi H, Kudo Y, Tashiro S, Yamamoto T, et al. Exploration of genetic basis underlying individual differences in radiosensitivity within human populations using genome editing technology. J Rad Res. (2018) 59(Suppl. 2):ii75-82. doi: 10.1093/jrr/rry007

32. Lobachevsky P, Leong T, Daly P, Smith J, Best N, Tomaszewski J, et al. Compromized DNA repair as a basis for identification of cancer radiotherapy patients with extreme radiosensitivity. Cancer Lett. (2016) 383:212-9. doi: 10.1016/j.canlet.2016.09.010

33. Berthel E, Ferlazzo ML, Devic C, Bourguignon M, Foray N. What does the history of research on the repair of DNA double-strand breaks tell us?A comprehensive review of human radiosensitivity. Int J Mol Sci. (2019) 20:5339. doi: 10.3390/ijms20215339

34. Foray N, Bourguignon $M$, Hamada N. Individual response to ionizing radiation. Mutat Res. (2016) 770:369-86. doi: 10.1016/j.mrrev.2016.09.001

35. Seibold P, Webb A, Aguado-Barrera ME, Azria D, Bourgier C, Brengues $\mathrm{M}$, et al. REQUITE: a prospective multicentre cohort study of patients undergoing radiotherapy for breast, lung or prostate cancer. Radiother Oncol. (2019) 138:59-67. doi: 10.1016/j.radonc.2019.04.034

36. Rosen EM, Fan S, Goldberg ID, Rockwell S. Biological basis of radiation sensitivity. Part 2: cellular and molecular determinants of radiosensitivity. Oncology (Huntingt). (2000) 14:741-57.

37. Jalal S, Earley JN, Turchi JJ. DNA repair: from genome maintenance to biomarker and therapeutic target. Clin Cancer Res. (2011) 17:697384. doi: 10.1158/1078-0432.CCR-11-0761

38. Chistiakov DA, Voronova NV, Chistiakov PA. Genetic variations in DNA repair genes, radiosensitivity to cancer and susceptibility to acute tissue reactions in radiotherapy-treated cancer patients. Acta Oncol. (2008) 47:80924. doi: 10.1080/02841860801885969

Conflict of Interest: The authors declare that the research was conducted in the absence of any commercial or financial relationships that could be construed as a potential conflict of interest.

Copyright (C) 2021 Alsbeih, Al-Harbi, Ismail and Story. This is an open-access article distributed under the terms of the Creative Commons Attribution License (CC BY). The use, distribution or reproduction in other forums is permitted, provided the original author(s) and the copyright owner(s) are credited and that the original publication in this journal is cited, in accordance with accepted academic practice. No use, distribution or reproduction is permitted which does not comply with these terms. 